INSTRUMENTE DER NEUZEIT 
ENGELHARD WEIGL

\section{INSTRUMENTE DER NEUZEIT}

Die Entdeckung der modernen Wirklichkeit 


\section{Für Ewe}

CIP-Titelaufnahme der Deutschen Bibliothek

Weigl, Engelhard:

Instrumente der Neuzeit : die Entdeckung der modernen Wirklichkeit / Engelhard Weigl. - Stuttgart : Metzler, 1990 ISBN 978-3-476-00711-7

ISBN 978-3-476-00711-7

ISBN 978-3-476-03323-9 (eBook)

DOI 10.1007/978-3-476-03323-9

Dieses Werk einschließlich aller seiner Teile ist urheberrechtlich geschützt. Jede Verwertung außerhalb der engen Grenzen des Urheberrechtsgesetzes ist ohne

Zustimmung des Verlages unzulässig und strafbar.

Das gilt insbesondere für Vervielfältigungen, Übersetzungen, Mikroverfilmungen und die Einspeicherung und Verarbeitung in elektronischen Systemen.

(C) 1990 Springer-Verlag GmbH Deutschland

Ursprünglich erschienen bei J.B. Metzlersche Verlagsbuchhandlung und Carl Ernst Poeschel Verlag GmbH in Stuttgart 1990 


\section{INHALT}

\section{Vorwort 7}

\section{Einleitung 9}

Wissenschaftliche Instrumente: Warum so spät? 9 Instrumente im Prozeß neuzeitlichen Selbstbewußtseins 9

Wissenschaft und die Krise des 17. Jahrhunderts 18 Über das Spannungsverhältnis von Wissenschaft und Technik 20

Das Teleskop und das Auge 25

Galilei und das Teleskop 25

Das Teleskop und das Auge 32

Reflexive Teleskopie

(Galilei, Hannah Arendt, Hans Blumenberg) 37

Das Zyklotron des 17. Jabrbunderts.

Otto von Guericke als Erfinder der Luftpumpe 53

Die technischen Voraussetzungen der Luftpumpe 53

Ikonographie der Luftpumpe 59

Theologie des leeren Raumes 64

Maxima in minimis - Die Erfindung des Mikroskops 68

Der langsame Durchbruch zu einer neuen Welt 68

Robert Hooke und Antoni Leeuwenhoek 73

Das Buch der Natur gegen das Buch der Bücher 79

Universalisierung als Problem - oder die langsame Erfindung des Thermometers 86

Schwierigkeiten bei der ersten Vermessung eines Winters 88

Die erste reproduzierbare Thermometer:

Roemer und Fahrenheit 96 
Die Wiedergewinnung des Paradieses.

Utopie und Instrumentenentwicklung 101

Endzeiterwartung und die Erneuerung der Wissenschaften 101

Skeptische Anthropologie und technische Utopie 106

Die Welt als Uhrwerk. Die Funktion einer Metapher in der früben Neuzeit 114

Epochen der Uhrenentwicklung 114

Die Uhr als Metapher 123

Genauigkeit als Problem - oder die Suche nach dem Längengrad 133

Das Problem der Standortbestimmung auf See 133

Das Problem der Genauigkeit 141

"In der Nähe ist alles nicht wahr" 150

Die Vermessung einer Nation - oder die Triangulation des terrestrischen Raumes 155

Die Anfänge der modernen Kartographie 155

Technische und institutionelle Voraussetzungen 157

Die Herstellung des Rahmens (1668-1718) 161

Die Vollendung der ersten Karte (1730-1744) 166

Ein Instrument gegen die Angst. Die Verbreitung des Blitzableiters im 18. Jabrbundert 175

Zwei Fallgeschichten 175

Theologischer Widerstand gegen den Blitzableiter 181

Das Gewitter als ästhetischer Gegenstand 186

Die Depotenzierung Gottes 191

Verabschiedung des 18. Jahrhunderts 197

Alexander von Humboldt: Zwischen Weimar und Paris 201

Erfahrungswissen versus Spekulation 201

Reisevorbereitungen 206

"Naturgemälde« 211

Vom reisenden Forschungsinstitut zur internationalen

Zusammenarbeit der Forschungsinstitute 215

Anmerkungen 227

)6( 


\section{VORWORT}

Diese Arbeit entstand im wesentlichen im Laufe des Jahres 1988, und wurde in monatlichen Essays von Januar bis Dezember 1988 in der japanischen Philosophiezeitschrift Gendai-shisou. Revue de la pensée d' aujourd' bui veröffentlicht. Die Vorarbeiten zu ihr liegen jedoch weit zurück. In einem Seminar über Hermann Samuel Reimarus 1968 an der Ruhruniversität Bochum machte Prof. Hans Blumenberg die für uns überraschende Bemerkung, daß der Blitzableiter ein lohnendes Thema sei. Er war sich allerdings nicht sicher, ob das Thema auch für eine Dissertation ausreichen würde, zu weit war der Gegenstand von dem entfernt, was traditionellerweise das Fach Philosophie ausmacht. Doch neben meiner Arbeit an der Doktorarbeit über Jean Paul (Aufklärung und Skeptizismus. Untersuchungen über Jean Pauls Frühwerk. Hildesheim 1980) begann mich das Thema immer mehr zu faszinieren, und ich sammelte in den Bibliotheken Material dazu. So abseitig das Thema auf den ersten Blick anmutete, so schien es mir doch auf eine anschauliche Weise in das Zentrum der Intention der Aufklärung zu führen, so wie es im ersten Satz der "Dialektik der Aufklärung " von Horkheimer und Adorno definiert wird : "Seit je hat Aufklärung im umfassendsten Sinn fortschreitenden Denkens das Ziel verfolgt, von den Menschen die Furcht zu nehmen und sie als Herren einzusetzen." Allerdings das "Aber « mit dem die Autoren der "Dialektik der Aufklärung « ihren zweiten Satz einleiten, tritt in der vorliegenden Studie zurück. Zu deutlich liegt es uns heute vor Augen, so daß es die historische Analyse eher zu verstellen droht, als bei ihr behilflich zu sein.

Das Material zu dieser Studie blieb lange liegen, andere Projekte schoben sich immer wieder dazwischen. Erst auf den langen Autofahrten von Suginamiku nach Chiba (Tokyo) zu den Studios der Hoso-Universität wurde das Thema in Gesprächen mit Prof. Tsuji wieder lebendig. Er schlug dann auch vor, es für das Lehrprogramm der Universität zu benutzen, und so wurde die erste Fassung in einem Interview mit Prof. Kenichi Mishima realisiert. Der wichtigste Schritt zu diesem Buch kam dann auch von Prof. Mishima. Er machte 1987, 
ein Dreivierteljahr vor meiner Abreise nach Australien, den Vorschlag, den Aufsatz über "Entzauberung durch Wissenschaft", der jetzt unter dem Titel »Ein Instrument gegen die Angst « erscheint, zu einer Serie für die Zeitschrift Gendai-shisou unter dem Titel "Instrumente der Neuzeit « zu erweitern, und er erklärte sich gleichzeitig dazu bereit, die Serie zu übersetzen. So verlockend ich damals den Vorschlag fand, ich ahnte noch nicht, auf welch ein Abenteuer ich mich damit einließ; fern von den europäischen Bibliotheken und während des Umzugs von Japan nach Australien mußte unter dem Druck der Serie die Artikelfolge termingerecht fertiggestellt werden.

In der Zwischenzeit wurden jedoch die einzelnen Artikel durchgesehen und zum Teil erheblich erweitert. Doch die Anlage des Buches bleibt geprägt vom Gesetz der Serie, das heißt, die einzelnen Kapitel können auch für sich gelesen werden. Auch manche Wiederholungen ließen sich - ohne die Systematik der Kapitel zu tangieren - nicht vermeiden.

$\mathrm{Zu}$ danken habe ich zuerst den gut sortierten Bibliotheken der Universität Tokyo (Kyoyo Gakka) und der Universität Adelaide, denn Bibliotheken sollen nicht nur das bereitstellen, was der Leser sucht, sie sollen ebenso auf Wichtiges aufmerksam machen, von dem der Suchende noch nichts weiß. Herr Kiichiro Oishi in Tokyo und Herr Gerhard Kluchert in Berlin haben mir geholfen, wichtige Bücher und Aufsätze zu besorgen, die mir in Australien unzugänglich waren. Frau Ewe Buck-Weigl und Herr Prof. Dr. Anthony Stephens haben mir bei Übersetzungen aus dem Französischen und Englischen geholfen. Doch gilt mein größter Dank Herrn Prof. Mishima : ohne ihn gäbe es das vorliegende Buch nicht.

$34^{\circ} 57^{\prime} 0^{\prime \prime}$ südlicher Breite $138^{\circ} 38^{\prime} 0^{\prime \prime}$ östlicher Länge

Freitag, den 11. Mai 1990 